\title{
PRODUCTS OF ARCWISE CONNECTED SPACES ${ }^{1}$
}

\author{
B. LEHMAN
}

\begin{abstract}
It is proved that the arbitrary product of arcwise connected spaces is arcwise connected.
\end{abstract}

Introduction. By an arc we mean a Hausdorff continuum with at most 2 noncut points, called the end points of the arc. A space $S$ is said to be arcwise connected if whenever $x, y \in S$, then $x$ and $y$ are the end points of some arc in $S$. It is well known (see, for instance, [4, Theorems 28.8 and 28.13]) that a nondegenerate metric continuum $A$ is an arc if and only if $A$ is homeomorphic to $[0,1]$. Since a metrizable product of arcs is a compact, connected and locally connected metric space, it follows [4, Theorem 31.2] that a metrizable product of arcs is arcwise connected. However, examples have been constructed by S. Mardesic [2] and [3] and J. L. Cornette and B. Lehman [1] of locally connected Hausdorff continua which are not arcwise connected. Thus the above argument will not suffice for a nonmetrizable product of arcs, even if each factor space is metrizable. In this paper we show that the arbitrary product of arcwise connected spaces is arcwise connected.

Lemma Let $\left\{X_{\alpha}: \alpha \in \mathscr{A}\right\}$ be a collection of nondegenerate arcs, and let $X$ denote the product space of this collection. If the end points of $X_{\alpha}$ are $a_{\alpha}$ and $b_{\alpha}$, then there is an arc in $X$ from $f$ to $g$ where $f$ is that point for which $f(\alpha)=a_{\alpha}$ and $g$ is that point for which $g(\alpha)=b_{\alpha}$.

Proof. Let $\leqq$ be a well-ordering of $\mathscr{A}$, and let "l" denote the first element of $\mathscr{A}$, and " $\alpha+1$ " the successor of $\alpha$ in $\mathscr{A}$. For each $\alpha \in \mathscr{A}$, define the "edge" $A_{\alpha}$ of $X$ and points $f_{\alpha}$ and $g_{\alpha}$ of $X$ as follows:

$$
\begin{aligned}
& A_{\alpha}=\left\{h \in X: h(\beta)=b_{\beta}, \beta<\alpha ; h(\beta)=a_{\beta}, \alpha<\beta\right\}, \\
& f_{\alpha}(\beta)=b_{\beta}, \quad \beta<\alpha, \quad g_{\alpha}(\beta)=b_{\beta}, \quad \beta \leqq \alpha, \\
& =a_{\beta}, \quad \beta \geqq \alpha ; \quad=a_{\beta}, \quad \beta>\alpha .
\end{aligned}
$$

Received by the editors May 4, 1973.

AMS (MOS) subject classifications (1970). Primary 54B10, 54F05, 54F20.

Key words and phrases. Arc, arcwise connected, product space.

1 The results in this paper are contained in the author's doctoral dissertation written under the supervision of J. L. Cornette of Iowa State University.

(c) American Mathematical Society 1974 
We show that for each $\alpha \in \mathscr{A}$, the following statements are satisfied:

(a) $f_{1}=f$, and $g_{\alpha}=f_{\alpha+1}$;

(b) $A_{\alpha} \cap A_{\alpha+1}=\left\{g_{\alpha}\right\}$;

(c) $A_{\alpha}$ is an arc homeomorphic to $X_{\alpha}$ in $X$ and the end points of $A_{\alpha}$ are $f_{\alpha}$ and $g_{\alpha}$;

(d) if $\alpha, \gamma \in \mathscr{A}$ and $\alpha+1<\gamma$, then $A_{\alpha} \cap A_{\gamma}=\varnothing$;

(e) $\bigcup_{\beta<\alpha} A_{\beta} \cup\left\{f_{\alpha}\right\}$ is an arc in $X$ with end points $f$ and $f_{\alpha}$. Further, $\bigcup_{\alpha \in \mathscr{A}} A_{\alpha} \cup\{g\}$ is an arc in $X$ with end points $f$ and $g$.

It is immediate from the definitions that (a) and (b) are satisfied. If we define a function $\theta: X_{\alpha} \rightarrow X$ by

$$
\begin{aligned}
{[\theta(x)](\delta) } & =b_{\delta}, & & \delta<\alpha, \\
& =x, & & \delta=\alpha, \\
& =a, & & \delta>\alpha,
\end{aligned}
$$

then $\theta$ is a homeomorphism from $X_{\alpha}$ onto $A_{\alpha}$ such that $\theta\left(a_{\alpha}\right)=f_{\alpha}$, and $\theta\left(b_{\alpha}\right)=g_{\alpha}$, so (c) is satisfied. Now if $\alpha, \gamma \in \mathscr{A}$, with $\alpha+1<\gamma$, and $h \in A_{\alpha}$, then $h(\alpha+1)=a_{\alpha+1} \neq b_{\alpha+1}$. If $h \in A_{\gamma}$, then $h(\alpha+1)=b_{\alpha+1}$, and it follows that $A_{\alpha} \cap A_{\gamma}=\varnothing$. Thus (d) is satisfied.

We now proceed to prove (e) by induction on the well-ordered set $\mathscr{A}$. Let $I(\beta)$ be the statement: $\bigcup_{\alpha<\beta} A_{\alpha} \cup\left\{f_{\beta}\right\}$ is an arc in $X$ with end points $f$ and $f_{\beta}$.

If $\beta=1$, then $\bigcup_{\alpha<\beta} A_{\alpha} \cup\left\{f_{1}\right\}=\{f\}$ so it is an (degenerate) arc with end points $f$ and $f_{1}$. Suppose that for some $\beta \in \mathscr{A}, 1<\beta$, that we have shown that $I(\alpha)$ holds for all $\alpha<\beta$. We consider two cases.

Case 1. $\beta=\gamma+1$ for some $\gamma \in \mathscr{A}$. By the induction hypothesis $\bigcup_{\alpha<\gamma} A_{\alpha} \cup\left\{f_{\gamma}\right\}$ is an arc in $X$ with end points $f$ and $f_{\gamma}$, and we have shown that $A_{\gamma}$ is an arc in $X$ with end points $f_{\gamma}$ and $g_{\gamma}=f_{\gamma+1}=f_{\beta}$. If $h \in \bigcup_{\alpha<\gamma} A_{\alpha} \cap$ $A_{\gamma}$, then it follows from (d) that $\gamma=\delta+1$ for some $\delta \in \mathscr{A}$ and $h \in A_{\delta} \cap A_{\gamma}$. It then follows from (a) and (b) that $h=g_{\delta}=f_{\delta+1}=f_{\gamma}$ and that $\left(\bigcup_{\alpha<\gamma} A_{\alpha} \cup\right.$ $\left.\left\{f_{\gamma}\right\}\right) \cap A_{\gamma}=\left\{f_{\gamma}\right\}$. We have then that $\bigcup_{\alpha<\gamma} A_{\alpha} \cup\left\{f_{\gamma}\right\}$ and $A_{\gamma}$ are arcs which meet in a single point, $f_{\gamma}$, and that $f_{\gamma}$ is an end point of each. Thus their union is an arc with end points $f_{1}$ and $g_{\gamma}=f_{\beta}$. That is $\bigcup_{\alpha \leqq \gamma} A_{\alpha} \cup\left\{f_{\beta}\right\}$ is an arc with end points $f$ and $g_{\gamma}=f_{\beta}$.

Case 2. $\beta$ has no immediate predecessor in $\mathscr{A}$. There are four steps in the argument.

(1) $\bigcup_{\alpha<\beta} A_{\alpha}$ is connected;

(2) If $h \in X$ and $h \notin \bigcup_{\alpha<\beta} A_{\alpha} \cup\left\{f_{\beta}\right\}$, then $h$ is not a limit point of $\bigcup_{\alpha<\beta} A_{\alpha}$;

(3) $f_{\beta}$ is a limit point of $\bigcup_{\alpha<\beta} A_{\alpha}$, so $\bigcup_{\alpha<\beta} A_{\alpha} \cup\left\{f_{\beta}\right\}$ is a continuum and $f_{\beta}$ is not a cut point of $\bigcup_{\alpha<\beta} A_{\alpha} \cup\left\{f_{\beta}\right\}$;

(4) $f$ is not a cut point of $\bigcup_{\alpha<\beta} A_{\alpha} \cup\left\{f_{\beta}\right\}$, and if $h \in \bigcup_{\alpha<\beta} A_{\alpha}$ and $h \neq f$, 
then $h$ is a cut point of $\bigcup_{\alpha<\beta} A_{\alpha} \cup\left\{f_{\beta}\right\}$; thus $\bigcup_{\alpha<\beta} A_{\alpha} \cup\left\{f_{\beta}\right\}$, is an arc with end points $f$ and $f_{\beta}$.

Proof of (1). By the induction hypothesis, $\bigcup_{\alpha<\beta} A_{\alpha}=\bigcup_{\gamma<\beta}\left(\bigcup_{\alpha<\gamma} A_{\alpha}\right)$ is the union of connected sets each of which contains $f$ and is therefore connected.

Proof of (2). For each $\alpha \in \mathscr{A}$ let $P_{\alpha}$ be the projection map of $X$ onto $X_{\alpha}$. Suppose that $h \in X-\left[\bigcup_{\alpha<\beta} A_{\alpha} \cup\left\{f_{\beta}\right\}\right]$. Let $\gamma$ be the first member of $\mathscr{A}$ such that $h(\gamma) \neq f_{\beta}(\gamma)$. If $\beta \leqq \gamma$ then $f_{\beta}(\gamma)=a_{\gamma} \neq h(\gamma)$ and $P_{\gamma}^{-1}\left(X_{\gamma}-\left\{a_{\gamma}\right\}\right)$ is open in $X$, contains $h$ and misses $\bigcup_{\alpha<\beta} A_{\alpha} \cup\left\{f_{\beta}\right\}$. Suppose then that $\gamma<\beta$. Then if $\delta<\gamma, h(\delta)=b_{\delta}$ and $h(\gamma) \neq b_{\gamma}$. If for all $\varepsilon \in \mathscr{A}$ such that $\gamma<\varepsilon, h(\varepsilon)=a_{\varepsilon}$, then $h \in A_{\gamma}$, contrary to assumption. Thus for some $\varepsilon \in \mathscr{A}, \gamma<\varepsilon$ and $h(\varepsilon) \neq a_{\varepsilon}$. It now follows from the definition of the sets $A_{\alpha}$ that the open set $P_{\gamma}^{-1}\left(X_{\gamma}-\left\{b_{\gamma}\right\}\right) \cap P_{\varepsilon}^{-1}\left(X_{\varepsilon}-\left\{a_{\varepsilon}\right\}\right)$ contains $h$ and misses $\bigcup_{\alpha<\beta} A_{\alpha}$. Thus $h$ is not a limit point of $\bigcup_{\alpha<\beta} A_{\alpha}$.

Proof of (3). We consider the net $\left\{g_{\alpha}\right\}_{\alpha<\beta}$. For all $\delta \in \mathscr{A}$, the net $\left\{P_{\delta}\left(g_{\alpha}\right)\right\}_{\alpha<\beta}$ converges to $P_{\delta}\left(f_{\beta}\right)$. For if $\beta \leqq \delta$, then for all $\alpha<\beta, g_{\alpha}(\delta)=$ $a_{\delta}=f_{\beta}(\delta)$; and if $\delta<\beta$, then since $\beta$ has no immediate predecessor, there is a $\gamma \in \mathscr{A}$, such that $\delta<\gamma<\beta$ and if $\gamma \leqq \varepsilon<\beta$, then $P_{\delta}\left(g_{\varepsilon}\right)=g_{\varepsilon}(\delta)=b_{\delta}=f_{\beta}(\delta)$. It follows that the net $\left\{g_{\alpha}\right\}_{\alpha<\beta}$ converges in $X$ to $f_{\beta}$. It now follows immediately from (1) that $f_{\beta}$ is not a cut point of $\bigcup_{\alpha<\beta} A_{\alpha} \cup\left\{f_{\beta}\right\}$.

Proof of (4). Since $\bigcup_{\alpha<\beta} A_{\alpha}-\{f\}=\bigcup_{\gamma<\beta}\left[\bigcup_{\alpha<\gamma} A_{\alpha}-\{f\}\right]$ is a union of the connected sets $\bigcup_{\alpha<\gamma} A_{\alpha}-\{f\}$ each of which contains $f_{2}, \bigcup_{\alpha<\beta} A_{\alpha}-$ $\{f\}$ is connected.

Now suppose that $h \in \bigcup_{\alpha<\beta} A_{\alpha}, h \neq f$. Let $\alpha^{*}$ be the first member of $\mathscr{A}$ such that $h \in A_{\alpha^{*}}$, and let $Y_{\alpha^{*}}, Z_{\alpha^{*}}$ be the subarcs in $X_{\alpha^{*}}$ (one possibly degenerate) with end points $a_{\alpha^{*}}, h\left(\alpha^{*}\right)$ and $h\left(\alpha^{*}\right), b_{\alpha^{*}}$, respectively. For each $\alpha \in \mathscr{A}$, define $S_{\alpha}$ and $T_{\alpha}$ as follows:

$$
\begin{aligned}
& S_{\alpha}=\left\{b_{\alpha}\right\}, \quad \alpha<\alpha^{*}, \quad T_{\alpha}=X_{\alpha}, \quad \alpha<\alpha^{*}, \\
& =Z_{\alpha^{*}}, \quad \alpha=\alpha^{*}, \quad=Y_{\alpha^{*}}, \quad \alpha=\alpha^{*}, \\
& =X_{\alpha}, \quad \alpha^{*}<\alpha, \quad=\left\{a_{\alpha}\right\}, \quad a^{*}<\alpha .
\end{aligned}
$$

Let $S$ and $T$ be the product spaces respectively of the collections $\left\{S_{\alpha}\right.$ : $\alpha \in \mathscr{A}\}$ and $\left\{T_{\alpha}: \alpha \in \mathscr{A}\right\}$. Then $S$ and $T$ are closed in $X$ and $S \cap T=\{h\}$. Now $f \in T, f_{\beta} \in S$, and $\bigcup_{\alpha<\beta} A_{\alpha} \cup\left\{f_{\beta}\right\} \subset S \cup T$. It follows that ([( $\cup_{\alpha<\beta} A_{\alpha} \cup$ $\left.\left.\left.\left\{f_{\beta}\right\}\right)-\{h\}\right] \cap S,\left[\left(\cup_{\alpha<\beta} A_{\alpha} \cup\left\{f_{\beta}\right\}\right)-\{h\}\right] \cap T\right)$ is a separation of $\left(\bigcup_{\alpha<\beta} A_{\alpha} \cup\right.$ $\left.\left\{f_{\beta}\right\}\right)-\{h\}$, so $h$ is a cut point of $\bigcup_{\alpha<\beta} A_{\alpha} \cup\left\{f_{\beta}\right\}$. Thus $I(\beta)$ is established and statement (e) follows.

By argument similar to that in the induction step of the proof of (e), it follows that $\bigcup_{\alpha \in \mathscr{A}} A_{\alpha}$ is connected; that if $h \in X-\bigcup_{\alpha \in \mathscr{A}} A_{\alpha}$ and $h \neq g$, then $h$ is not a limit point of $\bigcup_{\alpha \in \mathscr{A}} A_{\alpha}$; that the net $\left\{g_{\alpha}\right\}_{\alpha \in \mathscr{A}}$ converges in $X$ to $g$, and that $f$ and $g$ are the only noncut points of $\bigcup_{\alpha \in \mathscr{A}} A_{\alpha} \cup\{g\}$. Thus $\bigcup_{\alpha \in \mathscr{A}} A_{\alpha} \cup\{g\}$ is an arc in $X$ with end points $f$ and $g$. 
THEOREM. If $\left\{Y_{\alpha}: \alpha \in \mathscr{A}\right\}$ is a collection of arcwise connected spaces, and $Y$ is the product space of the collection, then $Y$ is arcwise connected.

Proof. Let $f, g$ be points of $Y$. If $f=g$, there is nothing to prove, so assume that $f \neq g$. Let $\mathscr{A}^{*}=\{\alpha \in \mathscr{A}: f(\alpha) \neq g(\alpha)\}$. For each $\alpha \in \mathscr{A}^{*}$, let $X_{\alpha}$ be an arc in $Y_{\alpha}$ with end points $a_{\alpha}=f(\alpha)$ and $b_{\alpha}=g(\alpha)$. Let $f^{*}$ and $g^{*}$ be the restrictions to $\mathscr{A}^{*}$ of $f$ and $g$ respectively, and let $X^{*}$ be the product space of the collection $\left\{X_{\alpha}: \alpha \in \mathscr{A}^{*}\right\}$. Then $\left\{X_{\alpha}: \alpha \in \mathscr{A}^{*}\right\}, f^{*}$ and $g^{*}$ satisfy the conditions of the Lemma, so there is in $X^{*}$ an arc $A$ with end points $f^{*}$ and $g^{*}$. Define a map $\theta: X^{*} \rightarrow Y$ by

$$
\begin{aligned}
{[\theta(h)](\alpha) } & =f(\alpha), & & \alpha \notin \mathscr{A}^{*}, \\
& =h(\alpha), & & \alpha \in \mathscr{A}^{*} .
\end{aligned}
$$

Then $\theta$ is a homeomorphism of $X^{*}$ onto $\theta\left(X^{*}\right)$ with $\theta\left(f^{*}\right)=f, \theta\left(g^{*}\right)=g$. Thus $\theta(A)$ is an arc in $Y$ with end points $f$ and $g$.

\section{REFERENCES}

1. J. L. Cornette and B. Lehman, Another locally connected Hausdorff continuum not connected by ordered continua, Proc. Amer. Math. Soc. 35 (1972), 281-284.

2. S. Mardešic, On the Hahn-Mazurkiewicz theorem in nonmetric spaces, Proc. Amer. Math. Soc. 11 (1960), 929-937. MR 22 \#8464.

3. - A locally connected continuum which contains no proper locally connected subcontinuum, Glasnik Mat. Ser III 2 (22) (1967), 167-178. MR 36 \#4526.

4. S. Willard, General topology, Addison-Wesley, Reading, Mass., 1970. MR 41 \#9173.

Department of Mathematics, University of Guelph, Guelph, Ontario, Canada 